Revista de Psicología Vol. 36 (1), 2018 (ISSN 0254-9247)

\title{
Locus de control en la elección de pareja: una validación etnopsicométrica
}

\author{
Joaquín Alberto Padilla-Bautista ${ }^{1}$, Rolando Díaz-Loving ${ }^{2}$, Isabel Reyes- \\ Lagunes $^{3}$, Christian Enrique Cruz-Torres ${ }^{4}$ y Nélida Padilla-Gámez ${ }^{5}$ \\ Universidad Nacional Autónoma de México ${ }^{1}$, Universidad de \\ Guanajuato-México ${ }^{4}$
}

En la actualidad pareciera que cada individuo es responsable de la elección de pareja, sin embargo, históricamente ha sido regulado por la cultura, familia y sociedad, quienes marcan las reglas, normas y conductas adecuadas para dicha elección. Esta investigación tuvo como propósito la construcción de una escala que analiza si una persona se considera a sí misma como responsable de la elección de su pareja; para ello se cuestiona la globalidad del proceso de locus de control. Un primer estudio, cualitativo, obtuvo indicadores usando ATLAS.ti, para obtener factores teóricos. Un segundo estudio corroboró los factores teóricos mediante un análisis de componentes principales, mostrando la existencia de los factores de: DestinoPredestinación, Poderosos del Microcosmos, y Control Interno, explicando con sus 17 ítems el $50.94 \%$ de varianza. Se concluye que el locus de control en la elección de pareja no es un continuo que se mueva entre internalidad y externalidad, sino que es multidimensional.

1 Licenciado en Psicología por la Universidad Nacional Autónoma de México (UNAM). Docente en la UNAM. Dirección postal: Avenida Universidad 3004, Cd. Universitaria, Mezzanine del edificio "D” cubículo 9 y 10, C.P. 04510, Ciudad de México. Contacto: nembrod@hotmail.com

2 PhD por la Universidad de Austin Texas. Profesor titular C de tiempo completo definitivo en la UNAM. Investigador nivel III del Sistema Nacional de Investigadores. Dirección postal: Avenida Universidad 3004, Cd. Universitaria, Mezzanine del edificio “D” cubículo 9 y 10 , C.P. 04510, Ciudad de México. Contacto: rdiazl@unam.mx

3 Doctora en Psicología por la UNAM. Profesora emérita de tiempo completo definitivo. Investigador nivel III del Sistema Nacional de Investigadores. Dirección postal: Avenida Universidad 3004, Cd. Universitaria, Mezzanine del edificio “D” cubículo 9 y 10, C.P. 04510, Ciudad de México. Contacto: lisabel@unam.mx

4 Doctor en Psicología por la UNAM. Profesor investigador en la Universidad de Guanajuato. Dirección postal: Av. Bosque Gomero 111-E. Fraccionamiento El Dorado. León, Guanajuato. C. P. 37547. Contacto: cassiel.79@gmail.com.

5 Doctora en Psicología por la UNAM. Profesor investigador asociado ” en la UNAM. Dirección postal: Avenida Universidad 3004, Cd. Universitaria, Mezzanine del edificio "D" cubículo 9 y 10, C.P. 04510, Ciudad de México. Contacto: nelidapadillag@gmail.com 
Palabras clave: locus de control, elección de pareja, validación psicométrica, ecuaciones estructurales, etnopsicometria.

\section{Locus of control in mate choice: Etno-Psychometric validation}

Today it seems as if each individual is responsible for choosing his/her mate. Historically, this process has been mainly regulated by culture, family and society, which make the rules and norms, and defines suitable behaviors in the mate selection process. This research aimed at constructing a scale to analyze if people consider themselves responsible for the choice of their partner and to what extent. A qualitative study using ATLAS.ti content analysis resulted in different theoretical factors. A second study sought to corroborate the theoretical factors using a principal component analysis, determining the following factors: Destiny-Predestination, Powerful Microcosm, and Internal Control factor, which explain 50.94\% of the variance with 17 items. The instrument shows that the locus of control in mate choice is not a continuum that moves between internality and externality but, rather, it is multidimensional. Keywords: Locus of control, mate choice, psychometric validation, structural equations, ethno psychometry

\section{Locus de controle na escolha do casal: uma validaçáo etnopsicometrica}

Hoje, parece que cada indivíduo é responsável pela escolha de seu parceiro, no entanto, tem sido historicamente regulado pela cultura, família e sociedade, que estabelecem regras, normas e comportamentos adequados a essa escolha. Esta pesquisa teve como objetivo construir uma escala que analise se uma pessoa se considera responsável pela escolha de seu parceiro; para isso, a globalidade do processo de locus de controle é questionada. Um primeiro estudo qualitativo, obteve indicadores usando ATLAS.ti, para obter fatores teóricos. Um segundo estudo corroborou os fatores teóricos através de uma análise de componentes principais, mostrando a existência dos fatores de Destinação-Predestinação, Poderosa do Microcosmo e Controle Interno, explicando com seus 17 itens 50.94\% de variância. Concluímos que o locus de controle na escolha do par não é um continuum que se move entre internidade e externalidade, mas é multidimensional.

Palavras chave: locus de controle, escolha de parceiro, validação psicométrica, equaçóes estruturais, etnopsicometria.

\section{Locus de contrôle dans le choix du couple: une validation ethnopsychométrique}

Actuellement, il semble que chaque individu est responsable du choix d'un couple, mais, historiquement, il a été réglementé par la culture, la famille et la société, qui marquent les règles, les normes et les comportements appropriés pour cette élection. Le but de cette recherche était la construction d'une échelle qui analyse si une personne se considère comme responsable du choix de son partenaire; pour cela, la globalité du processus de locus de contrôle est mise en question. Une première étude qualitative a obtenu des indicateurs en utilisant ATLAS.ti, pour obtenir des facteurs théoriques. Une deuxième étude a corroboré les facteurs théoriques au moyen d'une analyse des composantes principales, montrant l'existence des facteurs: Fate-Prédestination, Microcosme Puissant et Contrôle Interne, expliquant avec leurs 17 items les $50.94 \%$ de variance. Nous concluons que le locus de contrôle dans le choix du couple n'est pas un continuum qui se déplace entre l'internalité et l'externalité, mais qu'il est multidimensionnel. Mots-clés: locus de contrôle, choix du couple, validation psychométrique, équations structurelles, ethnopsychométrie. 
En la mayoría de las sociedades se ha privilegiado a la pareja como una forma de asegurar la continuidad de la especie y el progreso de la civilización, ya que esta institución cubre funciones biológicas, psicológicas y sociales sin las cuales la posibilidad de existencia de la especie humana no sería posible (Padilla-Gámez, 2012). Sin embargo, la búsqueda de pareja sentimental no siempre se ha manejado como conocemos hoy en día. Dentro del transcurso del tiempo y las culturas, la unión de dos personas ha sido definida como una unión sexual y económica, la cual ha sido regulada formalmente por los familiares y la sociedad (Murdock, 1949; Westermarck, 1921). Estas sanciones podrían deberse a que la formación de parejas tenía por objetivo satisfacer las necesidades políticas, sociales y económicas de las familias y no necesariamente a las de los individuos (Coontz, 2004, 2006). Por ello, se debe considerar que la elección de pareja no es únicamente instintiva, ya que, si bien es natural, se ha vuelto un proceso cada vez más complejo debido a los procesos psicológicos y socioculturales implicados (Díaz-Loving \& Sanchez, 2004).

Antes del siglo XIX, los individuos se veían imposibilitados de hacer una elección, o de contar con una relación de pareja totalmente libre, ya que ambos integrantes eran evaluados y elegidos por los padres con base en sus propios intereses, pues veían al matrimonio como un negocio donde las familias de los individuos que contraerían nupcias obtendrían beneficios (Antaki,2000). Es así que los futuros cónyuges podrían llegar hasta el momento del matrimonio incluso sin conocerse (Franco, 1995). Bredow, Cate y Huston (2008) refieren que no es sino hasta la década de 1920 que aparece un sistema de citas que adopta la idea de que las relaciones deben establecerse con base al amor y la atracción. Estos cambios trajeron consigo una ideología donde el matrimonio es una unión especial, a la que se llega una vez que se ha adquirido amplias experiencias en las relaciones (Cherlin, 2004). 
Ya en la actualidad y en la mayoría las culturas la elección de pareja recae principalmente en los propios individuos. Esta elección de pareja debe incluir una evaluación de los motivos de ambas partes, ya que de no considerarlos se pueden presentar situaciones que deriven en daños y vergüenza, así como repercusiones morales, sociales o económicas derivados de esa incongruencia (Bredow et al. 2008). Sin embargo, Buss, Schackelford, Kirkpatrick \& Larsen (2001) refieren que en la actualidad los individuos pueden tomar decisiones en el plano puramente personal sin restricciones familiares, culturales o morales. Es así que a lo largo de la historia se puede identificar que el poder de elección ha pasado a estar en manos de los propios individuos. Sin embargo, no se podría afirmar que es así para todas las regiones y/o culturas. Segalen (1992) indica que no se debe de caer en la simplificación de afirmar que, en momentos pasados, todos se casaban por interés y forzados por sus padres, mientras que hoy uno se casa por amor y elige sin ningún condicionamiento a su futuro esposo o esposa. Hoy, como ayer, los matrimonios no son ni totalmente libres ni totalmente impuestos.

Pareciera que el proceso de la elección de pareja a lo largo de la historia ha estado descrito por diferentes formas de afrontar la tarea pero, en la actualidad ¿cómo saber si un individuo se considera a sí mismo como responsable sobre la elección de su pareja? ¿Son los individuos quienes deciden, o se decide por ellos con qué pareja formar una relación? Estas preguntas no pueden ser respondidas sin analizar la responsabilidad con la que cuentan los individuos sobre sus decisiones y sin saber si se perciben como agentes de cambio del entorno en el que se encuentran. En otras palabras, es necesario analizar el foco de control, el cual permitiría conocer si un individuo se considera responsable de la elección de su pareja y con la capacidad de realizar la evaluación apropiada de los motivos de su elección, Bredow, Cate y Huston (2008) refieren que de no considerar los motivos de la futura pareja, así como los propios se pueden presentar situaciones peligrosas que deriven en daño y vergüenza derivados de la incongruencia de los motivos de una de las partes. 
Rotter (1966) refiere que cuando un sujeto se percibe así mismo como un agente que controla los reforzadores debido a sus características y habilidades personales, entonces tiene un sentido o locus de control interno. Por otro lado, los individuos desarrollan un locus de control externo cuando reciben un reforzador y este no es contingente a su conducta, sino que es causado por fuerzas que le rodean, percibiendo que quien controla estos refuerzos es algo ajeno a él. Así, el control de estos reforzadores recae en el mundo, la suerte, el destino y en "otros poderosos", pero no en el propio individuo. Es así que, dependiendo de sus experiencias de reforzamiento pasadas, los individuos desarrollan rasgos consistentes ya sean internos o externos. Desde esta postura, el constructo de locus de control debe entenderse como un constructo unidimensional, donde los polos de este continuo representan la máxima internalidad y la máxima externalidad, por lo que si un individuo tiende a ser más externo tenderá a ser menos interno (La Rosa, 1986).

Si bien, en su origen, el locus de control fue entendido como una solo dimensión donde los individuos se mueven a lo largo de un continuo, autores como Abrahamson, Schludermann y Schludermann (1973); Barling y Bolon (1980), mencionan que la unidimensionalidad de la escala de Rotter (1966) es bastante cuestionable, ya que dentro de la escala no solo contiene ítems que se relacionan con creencias personales, sino también con creencias ideológicas. Estos autores sugieren que la dimensión de Control Interno puede distinguirse entre dos dimensiones: una, donde el sujeto cree que tiene un nivel de control Individual (control personal), y otra donde el individuo considera que tiene el nivel de control que la mayoría de las personas de su sociedad posee (control Ideológico).

De la misma manera, Rotter (1975) reconoce posteriormente dos tipos de locus de control externo: los Defensivos, quienes consideran que el mundo es ordenado pero otras personas, a quienes consideran más poderosas, tienen el control del mundo. Y los Congruentes, quienes creen que el mundo es desordenado y que nadie puede cambiarlo, por lo que todo depende de la suerte y de otros sujetos más poderosos. 
Es esta falta de especificidad de la escala propuesta originalmente para medir internalidad-externalidad la que ocasionaría un bajo nivel predictivo, ya que no considera las múltiples dimensiones subyacentes.

Una explicación a la diferenciación de las dimensiones de locus de control puede estar relacionada a las diferencias culturales, ya que la cultura puede asignar un papel más o menos importante a la ideología. La percepción de las conductas que un individuo pueda controlar sería determinada por las normas que rigen su sociedad. De esta manera se espera que las premisas socioculturales dictaminen los parámetros de locus de control (Díaz-Loving \& Andrade, 1984). Uno de los instrumentos para medir locus de control con un impacto significativo de este constructo fue el desarrollado por Levenson (1974). Esta autora concibe al locus de control como multidimensional, por lo que el instrumento que desarrolla contempla tres subescalas: Control Interno, que se refiere a la creencia de que uno puede controlar su propia vida; $A z a r /$ Suerte, donde el control está relacionado con una percepción azarosa y desordenada del mundo; y Otros Poderosos, donde se da la creencia de que el mundo está ordenado y es predecible pero son otros sujetos quienes tienen el poder y control (La Rosa, 1986).

Díaz-Loving y Andrade (1984) han apoyado el argumento de la mutidimensionalidad del concepto de locus de control, encontrando en la población estudiantil infantil mexicana tres dimensiones: (a) Fatalista, donde el niño considera que no puede hacer algo para controlar su medio ambiente;(b) Afectiva, la cual refiere a situaciones en las que el nińo modifica su medio a través de sus relaciones afectivas, y (c) Instrumental, que describe situaciones donde el niño actúa directamente sobre el medio para conseguir lo que quiere.

La Rosa (1986), tomando en cuenta la multidimensionalidad del constructo y la importancia de considerar los parámetros culturales para su medición, junta las escalas propuestas por Rotter (1966) y Levenson (1974) obteniendo así cinco subescalas: (a) Fatalismo/suerte, que se refiere a creencias de un mundo no ordenado y los reforzadores dependen de elementos como el azar, la suerte o el destino; (b) Poderosos del macro cosmos o de enajenación política, que atribuye a personas 
alejadas al sujeto el control debido al poder con el que cuentan y cuya acción repercute en la vida del individuo; (c) Afectividad, cuando el sujeto cree consigue sus objetivos mediante relaciones afectivas de quienes les rodean; (d) Internalidad instrumental, en el caso en que el sujeto siente puede controlar su vida debido a su esfuerzo, trabajo y capacidad; y (e) Poderosos del micro cosmos, donde, al igual que en Poderosos del macro cosmos, el individuo percibe que individuos con poder son quienes controlan los reforzadores, sin embargo, estos son más cercanos al individuo, como pueden ser un jefe directo o los padres.

De esta manera, se puede observar que el constructo de locus de control no está conformado de forma polarizada como originalmente propuso Rotter (1966), sino que es un constructo multifactorial. Aunado a esto se debe de cuestionar sobre la globalidad del proceso y su especificidad, ya que posiblemente los individuos tiendan a asumirse como agentes cambiantes de su mundo solo en algún dominio específico de su vida, mientras que, en otro dominio, pueden identificarse como individuos que perciben las situaciones como impredecibles. Con base en los antecedentes teóricos presentados y, con la intención de obtener elementos para aplicar las dimensiones de locus de control al ámbito de la elección de pareja, el presente estudio tiene por objetivo poner a prueba la validez y consistencia interna de un instrumento diseñado para medir el locus de control en la elección de pareja. Se propone como hipótesis que el instrumento obtendrá indicadores adecuados de consistencia interna, reflejando la estructura teórica de locus de control como un constructo multidimensional y reflejando las características específicas del dominio de la elección de pareja.

Para ello se realizaron dos estudios. Uno, de carácter exploratorio, para obtener información de cómo viven los participantes la elección de pareja considerando en dónde está situado el locus de control de su toma de decisión. La información resultante de este primer estudio fue utilizada para la elaboración de reactivos que se pondrían a prueba en el segundo estudio, garantizando así que el instrumento obtenido es culturalmente válido. El segundo estudio analizó las respuestas al instrumento mediante la técnica de análisis factorial y la fórmula Alfa de 
Cronbach, obteniéndose evidencias de su validez de constructo y consistencia interna. Por último, se usaron los datos obtenidos del segundo estudio para llevar a cabo un modelamiento de ecuaciones estructurales, lo cual permitió realizar un análisis factorial confirmatorio y corroborar el correcto ajuste del modelo propuesto.

\section{Estudio 1}

\section{Método}

\section{Participantes}

Se entrevistó a 15 hombres y 13 mujeres de entre 18 y 34 años, con una media de 23 años. De la ciudad de México y área conurbada El $60.7 \%$ reportaron tener una relación de pareja. El $39 \%$ de los participantes reportaron tener una escolaridad máxima de preparatoria mientras que el resto se encontraba estudiando una licenciatura (e.g. ciencias de la tierra, medicina, odontología, ingeniera, mercadotecnia o administración).

\section{Medición}

Se realizó una revisión teórica de las definiciones conceptuales de locus de control, y tomando como partida las propuestas por Rotter (1966) y La Rosa (1986) se procedió a redactar una guía de entrevista semiestructurada. Estas preguntas fueron redactadas para saber dónde se deposita el foco de control en el proceso de elección de pareja (por ejemplo, ¿Quién consideras que es responsable de la elección de tu pareja? ¿Consideras que existen personas que se comportan de diferente manera? ¿Cómo son? ¿Cómo es la elección de estas personas?).

\section{Procedimiento}

Para la aplicación de la entrevista estructurada, con la finalidad de conocer el foco de control en la elección de pareja, se pidió la colabora- 
ción de estudiantes de la Universidad Nacional Autónoma de México, quienes invitaron a otros, cuyas edades estuvieran entre 18 y 22 años y entre 28 y 34 años de edad, a participar en el estudio. El único criterio de exclusión fue ser estudiante de psicología. Se escogieron estos rangos de edad ya que el grupo de 18 a 22 representa una etapa de vida donde los individuos se encuentran en el proceso de la elección y noviazgo, el segundo grupo de 28 a 32 representa a sujetos que ya han pasado el promedio de edad de matrimonio que es de 28 años (Instituto Nacional de Estadística, Geografía e Informática, 2013). Habiendo alcanzado el criterio de saturación teórica se contó, finalmente, con 28 participantes.

\section{Análisis de datos}

Las respuestas que brindaron los participantes se transcribieron en forma de afirmación y en primera persona (por ejemplo, "La decisión de quien será mi pareja no me corresponde”.) Estas afirmaciones, que refieren a situaciones donde la elección de pareja describe a diferentes tipos de locus de control, se emplearon como reactivos. Se revisó que la redacción de los reactivos fuera coherente. Se obtuvieron finalmente 47 reactivos. Este procedimiento permitió identificar qué ítems se ubicaban en un mismo continuo, que va desde "Me describe nada" a "Me describe mucho", opción de medición escogida para recuperar la proyección de los sujetos. Así mismo, se aseguró que existiera una unidimensionalidad en los reactivos, es decir, que solo existiera una sola idea por ítem. Los ítems obtenidos fueron analizados mediante el programa de Análisis cualitativo de datos textuales ATLAS.ti.

\section{Resultados}

Los reactivos analizados mediante este procedimiento permitieron obtener factores teóricos de locus de control en la elección de pareja. Los resultados se pueden ver en la Tabla 1. Posteriormente se descartaron todos los ítems que no se incluyeran en una categoría. 


\section{Tabla 1}

Factores teóricos del locus de control en la elección de pareja

\begin{tabular}{|c|c|c|}
\hline $\begin{array}{l}\text { Factores/ } \\
\text { Códigos }\end{array}$ & $\begin{array}{l}\text { Cantidad de } \\
\text { reactivos / } \\
\text { Fundamentos }\end{array}$ & Ejemplo de reactivo \\
\hline $\begin{array}{l}\text { Emocional } \\
\text { microcosmos }\end{array}$ & 1 & $\begin{array}{l}\text { No quiero decepcionar a mi familia al } \\
\text { escoger a la persona equivocada para mí. }\end{array}$ \\
\hline Macrocosmos & 2 & Estoy con mi pareja porque ella me eligió. \\
\hline $\begin{array}{l}\text { Control } \\
\text { emocional }\end{array}$ & 2 & $\begin{array}{l}\text { Cuando elijo pareja, me dejo llevar por } \\
\text { mis emociones, si estoy alegre busco a } \\
\text { alguien alegre si estoy triste elijo a alguien } \\
\text { triste. }\end{array}$ \\
\hline Presión social & 4 & $\begin{array}{l}\text { Mis familiares me presionan para tener } \\
\text { una relación de pareja. }\end{array}$ \\
\hline $\begin{array}{l}\text { Externalidad } \\
\text { despreocupada }\end{array}$ & 4 & $\begin{array}{l}\text { Si una relación de pareja se da qué bueno, } \\
\text { sino qué se le va a hacer. }\end{array}$ \\
\hline Externalidad & 4 & $\begin{array}{l}\text { Me gusta que me digan con quien iniciar } \\
\text { una relación, eso hace las cosas más } \\
\text { fáciles. }\end{array}$ \\
\hline Destino/ Dios & 9 & $\begin{array}{l}\text { Es el destino quien me dice con qué } \\
\text { persona voy a tener una relación. }\end{array}$ \\
\hline Internalidad & 10 & $\begin{array}{l}\text { Elijo a mi pareja bajo mis propios } \\
\text { criterios. }\end{array}$ \\
\hline Microcosmos & 11 & $\begin{array}{l}\text { Me gusta que mi familia escoja a la } \\
\text { persona con la que tengo que tener una } \\
\text { relación. }\end{array}$ \\
\hline
\end{tabular}

Estos factores teóricos fueron empleados en el segundo estudio, el cual permitió conocer mediante un análisis factorial exploratorio, qué reactivos pertenecían las dimensiones propuestas. 


\section{Estudio 2}

\section{Método}

\section{Participantes}

Empleando un muestreo por conveniencia en la ciudad de México y área conurbada se obtuvieron respuestas de 235 participantes, 113 hombres y 122 mujeres, entre los 18 y los 32 ańos con una media de 21.37 años. El $80 \%$ de la población reportó tener una relación de pareja al momento de la aplicación.

\section{Medición}

Se emplearon los 47 reactivos obtenidos del Estudio 1. Adicionalmente se redactaron las siguientes indicaciones que el participante tenía que leer para contestar a cada uno de los reactivos:

A continuación, se presentan descripciones breves que distintas personas dijeron sobre si mismas. Por favor, lea cada descripción y piense qué tanto usted se parece a cada una de ellas. Posteriormente marque el cuadro que usted considere apropiado. Entre más grande sea el cuadro, significa que esta afirmación lo describe más.

Las instrucciones presentadas tienen el propósito de disminuir el efecto de deseabilidad social en los participantes, ya que se tiene la hipótesis de se sentirán identificados con otras personas que son similares a ellos, además que de esta forma los individuos se proyectan con cada una de sus respuestas, lo que brinda una medición acerca de su personalidad.

Se empleó una escala tipo Likert pictórica de 5 puntos que va desde "Me describe nada" a "Me describe mucho". Se escogió esta forma de respuesta ya que se considera que la opción comúnmente aplicada de "Totalmente en desacuerdo" a "Totalmente de acuerdo" en realidad representa dos polos continuos, donde el participante responde al número tres con la intención de no estar en ninguna de las dos. Este formato de aplicación claramente es incongruente con 
la escala de medición continua, además de que facilita una regresión a la media de las respuestas, ya que los participantes escogen la casilla 3 con la intención de no estar en ninguna de las respuestas.

\section{Procedimiento}

Una vez desarrollados los ítems con las características antes mencionadas se solicitó la colaboración de estudiantes de la Universidad Nacional Autónoma de México, quienes a cambio de un punto en su calificación realizaron aplicaciones con sus familiares y conocidos. Los criterios de inclusión fueron los rangos de edad de 18 a 22 y de 28 a 32 y el criterio de exclusión que fueran estudiantes de psicología, esto con el propósito de tener una muestra más representativa de la cultura mexicana.

\section{Análisis de datos}

Se realizó una prueba de consistencia interna mediante un análisis de fiabilidad Alfa de Cronbach, se evaluó la correlación ítem-total; la correlación al cuadrado (varianza explicada) con los reactivos de la escala; y el valor de fiabilidad si se eliminaba el reactivo. Se eliminaron los reactivos que al tener menor relación con la escala disminuían el valor de Alfa.

\section{Resultados}

Se realizó un análisis de componentes principales con rotación ortogonal ya que las correlaciones entre ítems fueron entre .2 a .4. Se eliminaron los ítems que no se agruparan en un factor con cargas factoriales superiores a .4 , que se agruparan dentro de un factor teórico diferente al propuesto o que no se agrupara en un factor que tuviera por lo menos tres ítems.

En la solución final los eigenvalues superiores a 1 mostraron la existencia de cuatro factores. Esta solución convergió en cinco iteraciones y 
explica el $50.94 \%$ de la varianza. Los ítems presentan cargas factoriales superiores a .50 dentro de su factor y comunalidades mayores a .35 .

El instrumento final quedó conformado por 17 reactivos que se presentan en la Tabla 2. La prueba de esfericidad de Bartlett fue significativa (1211.18, $g l=136, p<.001)$, y el indicador de adecuación del tamaño de muestra Kaiser-Meyer-Olkin fue adecuado (KMO=.807). El Alfa del instrumento total fue de $\alpha=.68$.

\section{Tabla 2}

Ponderaciones de factores para el análisis factorial exploratorio del inventario de locus de control en la elección de pareja

\begin{tabular}{lccc}
\hline & $\begin{array}{c}\text { Poderosos del } \\
\text { microcosmos }\end{array}$ & $\begin{array}{c}\text { Destino/ } \\
\text { predestinac. }\end{array}$ & $\begin{array}{c}\text { Control } \\
\text { interno }\end{array}$ \\
\hline Rango & $1-5$ & $1-5$ & $1-5$ \\
Media & 1.33 & 2.26 & 4.28 \\
Desviación estándar & .53 & .98 & .70 \\
Sesgo & 2.43 & .69 & -1.23 \\
Varianza explicada & 19.89 & 17.27 & 13.68 \\
Alfa de Cronbach & Reactivo & \multicolumn{3}{c}{ Carga factorial } \\
\hline
\end{tabular}

1 Busco una pareja como la que quieren mis $\quad .756$ padres para mí.

2 La elección de mi pareja está determinada $\quad .728$ por lo que dicen mis amigos.

3 Solo puedo iniciar una relación de .719 $.135 \quad-.141$ noviazgo con alguien hasta que mis familiares aprueban a una persona.

4 La elección de mi pareja está determinada por lo que dice mi familia.

5 Para que inicie una relación de noviazgo con alguien, mis amigos tienen que aprobarla. 
Poderosos del Destino/ Control

microcosmos predestinac. interno

6 Necesito que personas cercanas a mí, aprueben a la persona con quien voy a iniciar una relación. Necesito que personas

cercanas a mí, aprueben a la persona con quien voy a iniciar una relación.

7 No me emparejo con alguien cuando mis padres me dicen que no me conviene.

8 Estoy con mi pareja porque el destino quería que fuera así.

9 Creo que el destino me unirá con la persona indicada.

10 El destino puso a mi pareja en mi camino.

11 Es el destino quien me dice con qué persona voy a tener una relación.

12 Creo que Dios pone a la persona adecuada en nuestro camino para tener una relación.

13 Yo soy el único responsable de encontrar una buena pareja.

$\begin{array}{lllll}14 & \text { Elijo a mi pareja bajo mis propios criterios. } & -.112 & -.156 & \mathbf{7 3 5}\end{array}$

15 No importa que me digan con quien debo de iniciar una relación, yo soy quien $\quad-.240$ decide con quien estar.

16 Aunque todos opinen, al final soy yo quien decide que pareja elegir.

17 Si yo quiero estar con una persona voy a estar ahí, sin importar lo demás.

Nota. Las cifras en negritas indican las cargas factoriales más altas. 
Con el propósito de conocer qué posibles relaciones se dan entre los factores se realizó una correlación de Pearson para hombres y mujeres. Los datos se muestran en la Tabla 3.

\section{Tabla 3}

Correlaciones entre los factores de la escala de locus de control

\begin{tabular}{lccc}
\hline & 1 & 2 & 3 \\
\hline 1. Destino/ Predestinación & - & .14 & $.30^{*}$ \\
2. Poderosos del microcosmos & $.26^{*}$ & - & -.14 \\
3. Control interno & -.02 & $-.51^{*}$ & - \\
\hline
\end{tabular}

*. Correlaciones significativas al .01

Nota. Las intercorrelaciones para las participantes mujeres $(n=122)$ se presentan sobre la diagonal y las intercorrelaciones para participantes hombres $(n=113)$ se presentan debajo de la diagonal

Posteriormente se llevó a cabo un análisis factorial confirmatorio mediante el programa AMOS 23, esto con el fin de contrastar la estructura factorial exploratoria. Se utilizó para la estimación de los parámetros de bondad de ajuste el método de máxima verosimilitud. Se emplean índices adicionales a la Chi cuadrada $\left(x^{2}\right)$ debido a que esta es muy sensible al tamańo de la muestra; a los supuestos de la curva normal, así como a la linealidad, multinormalidad y aditividad (Iraurgi, Sanz \& Martínez-Pampliega, 2009). Estos índices de ajuste, mencionados por Bentler, (1990); Bentler y Bonett (1980); Browne y Cudeck, (1993) y Lévy \& Varela (2008), se pueden ver en la Tabla 4. En la Figura 1 se muestra el modelo con los puntajes estandarizados. 


\section{Tabla 4}

Indices de ajuste esperados para un modelo de ecuaciones estructurales e indices obtenidos para el análisis factorial confirmatorio

Índice de ajuste

Esperado Obtenido

Chi-Cuadrado $x^{2}$

$>.05 \quad .001$

Discrepancia entre $\chi 2$ y grados de libertad; (CMIN/DF) $<5 \quad \mathbf{1 . 5 0}$

Índice de bondad de ajuste (GFI)

$.90-1 \quad .916$

Índice de ajuste ponderado (AGFI)

$.90-1$

Índice residual de la raíz cuadrada media (RMR)

Lo más cercano $\quad \mathbf{0 5 9}$

a 0

Error cuadrático media de aproximación (RMSEA)

$<.05$

.048

Índice de ajuste comparativo (CFI)

$.90-1 \quad \mathbf{9 3 3}$

Índice de ajuste normalizado (NFI)

$.90-1$

Índice no normalizado de ajuste (NNFI o TLI)

$.90-1$

.921

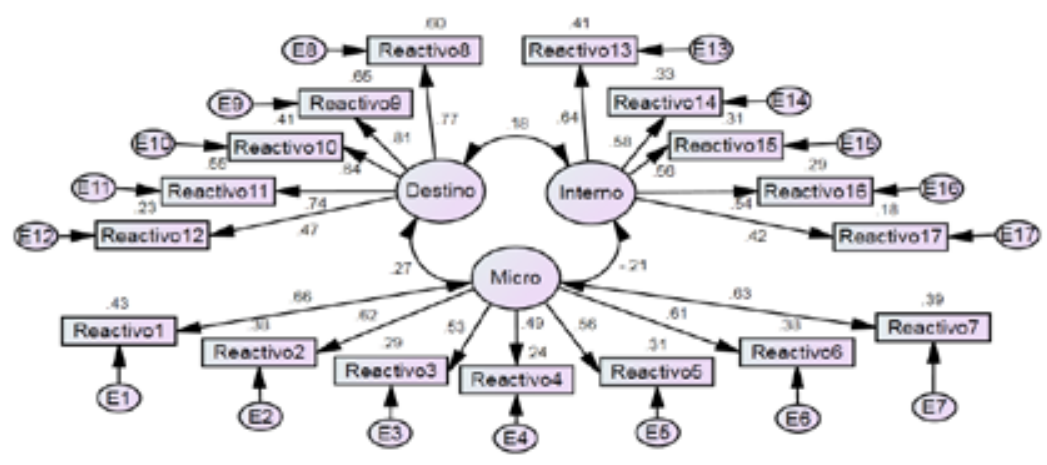

Figura 1. Modelo del análisis factorial confirmatorio.

$$
x^{2}=174.64, g l=116, p=.001
$$




\section{Discusión}

Los ítems que se incluyen en la escala final muestran una buena consistencia interna, el análisis factorial final cuenta con una buena varianza explicada. El análisis factorial confirmatorio mostró tener un ajuste adecuado. Estos resultados permiten afirmar que es posible contar con un instrumento de autorreporte para la medición del dominio específico del locus de control en la elección de pareja. Asimismo, permite demostrar que existe una manifestación independiente de cada uno de los estilos dentro del propio individuo. Los ítems muestran una correcta agrupación teórica dentro de cada uno de los factores obtenidos, los cuales a su vez son congruentes con la teoría antes expuesta, ya que los Poderosos del microcosmos equivalen al Control externo defensivo propuesto por Rotter (1975), a Otros poderosos propuesto por Levenson (1974), y a Poderosos del microcosmos propuestos por La Rosa (1986). Esto nos lleva a afirmar que este es un factor de locus de control externo donde los sujetos tienen la creencia de que existe un mundo ordenado y predecible; sin embargo, el control sobre la elección de su pareja está determinado por otros individuos, ya se necesita de la aprobación de los miembros cercanos al individuo - por ejemplo, familiares o amigos - para llevar a cabo la elección de pareja.

El factor Destino-Predestinación también muestra una congruencia teórica con el factor externo propuesto por Rotter (1975), con el de Azar/Suerte propuesto por Levenson (1974), y con el de Fatalismo/Suerte propuesto por (La Rosa, 1986). En este factor se tiene la creencia de que el mundo no es ordenado, por lo que el control de los reforzadores estaría relacionado con el destino, la suerte y Dios. Sin embargo, debe hacerse notar que este factor difiere con los propuestos a la teoría en el aspecto de que no está permeado por una connotación negativa, sino que pareciera tener un carácter romántico, ya que es el destino quien une al individuo con la persona adecuada para conformar una relación de pareja. Por último, se encuentra el factor Interno, también congruente con las escalas de Rotter (1966), Levenson (1974) y La Rosa (1986). En este factor se tiene la creencia de que son los propios 
individuos quienes pueden controlar la elección de su pareja, ya que se consideran capaces y los únicos responsables de realizar dicha elección. Si bien en la fase cualitativa se obtuvieron ítems que referían al Control Afectivo propuesto por La Rosa (1986), así como otros factores diferentes, estos reactivos no mostraron una agrupación coherente en el análisis de componentes principales por lo que fueron descartados.

Aunque el sesgo del factor de Poderosos del Microcosmos muestra que las calificaciones de los participantes tienden a ser menores, el análisis de componentes principales toma a este factor como el primero y el elemento que más varianza explica del fenómeno de locus de control en la elección de pareja. Esto puede explicarse a raíz de que la cultura mexicana está caracterizada por dar una mayor importancia a los vínculos interdependientes con otras personas, asignando más importancia a compartir temas personales y comunicarse (Sánchez \& Díaz-Loving, 1997), por lo que la toma de decisión de qué pareja elegir estará permeada por los círculos más cercanos. Los datos obtenidos por Padilla Gámez y Díaz-Loving (2013) corroboran estos datos, ya que refieren que la población mexicana percibe ciertos mandatos o premisas que son transmitidos directamente o indirectamente en su seno familiar. Por lo que estas premisas siguen ejerciendo cierto control en la forma en que las personas establecen relaciones diádicas.

Se propone como hipótesis que el factor de control interno puede tener una media de calificación más alta debido a que el cuestionario es un instrumento de autorreporte, que emplea el sistema de procesamiento central (Cacioppo \& Petty, 1982). Es decir, tiende a ser una actividad mental esforzada que demanda cálculos complejos y el sujeto, al estar consciente de sus respuestas, puede estar propenso a la deseabilidad social (Padilla-Bautista, Díaz-Loving \& Reyes-Lagunés, 2013). Si esto fuera cierto, los puntajes aquí observados podrían ser más bajos si se realiza un procedimiento en el cual se recojan calificaciones del sistema de pensamiento periférico, como pueden ser un test de actitudes implícitas (Briñol et al. 2002), o un test especial (Padilla-Bautista, DíazLoving \& Reyes-Lagunés, 2013), ya que este sistema tiende a operar de manera rápida y automática, con poco o ningún esfuerzo, y sin tener 
la sensación de control voluntario (Kahneman, 2012), por lo que las calificaciones estarían carentes de esta deseabilidad social.

Las correlaciones realizadas entre factores segmentadas por sexo, en los hombres se ve que tienden a contar con un mayor control interno, es decir, se asumen como agentes quienes pueden controlar la elección de su pareja, necesitarán menos de la aprobación de los miembros cercanos a él, sean estos amigos o familia. Así mismo, perciben que el control del microsmos está relacionado con el destino, por lo que el hecho de depositar la responsabilidad de la elección de la pareja en manos de familiares y amigos vuelve a la elección como algo impredecible y de lo cual no se tiene elección.

Para las mujeres se observa una correlación significativa entre el factor de Control Interno y el Destino-Predestinación. Esta correlación hace notar que la elección de pareja para las mujeres está bajo su control; sin embargo, debe existir influencia por parte del destino o Dios, el cual parece fungir como un cómplice para que se lleve a cabo la elección.

Cabe destacar que, si bien esta investigación refiere a la importancia de emplear medidas específicas, se debe de buscar mediante la réplica del estudio, la validez de estas dimensiones en otras culturas diferentes a la mexicana, y con ello poder reafirmar la expresión de locus de control en la elección de pareja aquí encontradas o bien complementarlas.

El instrumento obtenido de esta investigación es breve y práctico en su aplicación y permite obtener datos que soportan un amplio manejo de técnicas estadísticas, desde análisis de correlaciones, diferencias, modelos de regresión, e incluso modelos de ecuaciones estructurales.

Este artículo, además, pretende ser una guía para la construcción de un instrumento con medidas culturalmente relevantes, ya que propone tres fases compuestas por un estudio cualitativo y estudios psicométricos que permiten tener una validez de contenido del fenómeno que se está estudiando, así como evidencia empírica que corrobora la validez y confiabilidad del instrumento. 


\section{Referencias}

Abrahamson, D., Schludermann, S. \&Schludermann, E. (1973). Replication of dimensions of locus of control. Journal of Consulting and Clinical Psychology, 41, 320. https://doi.org/10.1037/ h0035121

Antaki, I. (2000). En el banquete de Platón: religión. Ciudad de México, México, México: Joaquín Mortiz.

Barling, J. \& Bolon, K. (1980). Multidimensional locus of control: the case of white South African student. Journal of Social Psychology, 111, 295-296. Recuperado el 27 de junio de 2016 de http:// web.business.queensu.ca/faculty/jbarling/Articles/1980\%20 Barling\%20Bolon.pdf

Bentler, P.M. (1990). Comparative fit indexes in structural models. Psychological Bulletin, 107, 238-246. http://dx.doi.org/10.1037/ 0033-2909.107.2.238

Bentler, P.M. \& Bonett, D.G (1980). Significance tests and goodness of fit in the analysis of covariance structures. Psychological Bulletin, 88, 588-606. http://dx.doi.org/10.1037/0033-2909.88.3.588

Bredow, C., Cate, R. \& Huston, T. (2008). Have We Met Before? A Conceptual Model of First Romantic Encounters. En S. Sprecher, A. Wenzel y J. Harvey (Eds.), Handbook of Relationship Initiation (pp. 3-28). New York: Psychology Press.

Briñol, P., Horcajo, J., Becerra, A., Falces, C. \& Sierra. B. (2002). Cambio de actitudes implícitas. Psicothema 14, 4 771-775.

Browne, M. W. \& Cudeck, R. (1993). Alternative ways of assessing model fit. En K. A. Bollen y J.S. Long (Eds.), Testing structural equation models (pp. 136-162). Newbury Park: Sage.

Buss, D.M., Shackelford, T.K., Kirkpatrick, L.A. \& Larsen, R.J. (2001). A half century of mate preferences: the cultural evolution of values. Journal of Marriage and Family, 63, 491-503. https://doi.org/10.1111/j.1741-3737.2001.00491.x 
Cacioppo, J. T. \& Petty, R. E. (1982). The need for cognition. Journal of Personality and Social Psychology, 42, 116-131. http://dx.doi. org/10.1037/0022-3514.42.1.116

Cherlin, A. (2004). The deinstitutionalization of American marriage. Journal of Marriage and Family, 66, 848-861. https:// doi:10.1111/j.00222445.2004.00058.x

Coontz, S. (2004). The world historical transformation of marriage. Journal of Marriage and Family, 66, 974-979. https://doi. org/10.1111/j.0022-2445.2004.00067.x

Coontz, S. (2006). Marriage, a history: How Love Conquered Marriage. New York: Viking.

Díaz-Loving, R. \& Andrade Palos, P. (1984). Una escala de locus de control para nińos mexicanos. Revista Interamericana de Psicologia, 18. 21-33.

Díaz-Loving. R. \& Sánchez Aragón, R. (2004). Psicología del amor: una visión integral de la relación de pareja. Ciudad de México, México: Porrúa.

Franco, C. (1995). La pareja: el arte de compartir la vida. Buenos Aires, Argentina: Salerno.

Instituto Nacional de Estadística, Geografía e Informática, INEGI. (2013). México.

Iraurgi,I. Sanz, M. \& Martínez-Pampliega, A. (2009). Adaptación y estudio psicométrico de dos instrumentos de pareja: índice de satisfacción matrimonial y escala de inestabilidad matrimonial. Revista IIPSI, 12(2), 177-192.

Kahneman, D. (2012). Pensar rápido, pensar despacio. México: Debate. La Rosa, J. (1986). Escalas de locus de control y autoconcepto:construcción y validación. Tesis de doctorado no publicada. Facultad de Psicología Universidad Nacional Autónoma de México.

Levenson, H. (1974). Activism and powerful others: Distinctions within the concept of internal-external control. Journal of Personality Assessment, 38, 377-383. http://dx.doi.org/10.1037/ h0035127 
Lévy, J. P. \& Varela, J. (2003). Análisis Multivariable para las Ciencias Sociales. Madrid: Pearson Educación.

Murdock, G.P. (1949). Social Structure. New York: The MacMillan Company.

Padilla-Bautista, J.A. Díaz-Loving, R. \& Reyes-Lagunes, I. (2013). Manchas de tinta y autoconcepto del mexicano: relación Etnopsicología entre lo consciente y lo inconsciente. Persona, 16, 11-27.

Padilla-Gámez, N. (2012). Estrategias de emparejamiento en población mexicana. Tesis doctoral no publicada. Facultad de Psicología, UNAM. México.

Padilla Gámez, N., Díaz-Loving, R. (2013). Premisas familiares y socioculturales del emparejamiento. Enseñanza e Investigación en Psicologia, 18(2), 249-262. Recuperado de http://www.redalyc. org/articulo.oa?id=29228336003

Rotter, J. B., (1966). Generalized expectancies for internal versus external control of reinforcement. Psychological monographs, 80(1), 1-28. http://dx.doi.org/10.1037/h0092976

Rotter, J.B. (1975). Some problems and misconceptions related to the construct of internal versus external control of reinforcement. Journal of Consulting and Clinical Psychology, 43, 56-67. https://doi.org/10.1037/h0092976

Sánchez Aragón, R. \& Díaz-Loving, R. (1997). Medición e implicaciones de la cercanía en el ciclo vital de la pareja. Revista de Psicología Social y Personalidad, 13, 1-18.

Segalen, M. (1992). Antropología histórica de la familia. Madrid: Taurus. Westermarck, E. (1921). The history of human marriage. Nueva York: Macmillan.

Recibido: 25 de noviembre, 2016 Revisado: 18 de setiembre, 2017 Aceptado: 18 de octubre, 2017 